\title{
Endoscopic Management of Colocutaneous Fistula After Percutaneous Endoscopic Gastrostomy
}

A 53-year-old man with a history of hypoxic brain damage and diabetes mellitus underwent insertion of a percutaneous endoscopic gastrostomy (PEG) tube because of difficulty in swallowing and accompanying malnutrition. At 2 weeks after the PEG procedure, he suddenly developed diarrhea and vomiting of feculent material, which was also found from PEG tube aspiration. An immediate fistulogram showed the tipp of the PEG tube in the transverse colon and a gastrocolic fistulous tract formation without peritoneal leakage. After 10 days of total parenteral nutrition and systemic antibiotics, a follow-up gastroscopy demonstrated complete healing of the gastrocolic fistula. After an enema had been administered, colonoscopy was undertaken in an attempt to remove the PEG tube. After the external PEG tube had been cut, and the internal tube had been pulled using a polypectomy snare, the 7-mm opening of the colocutaneous fistula was revealed (Figure 1). Considering the high risk of delayed wound healing and infection related to the patient's diabetes, we tried to close the fistulous opening endoscopically using a metallic clip. The open clip prong was easily applied to close the fis-

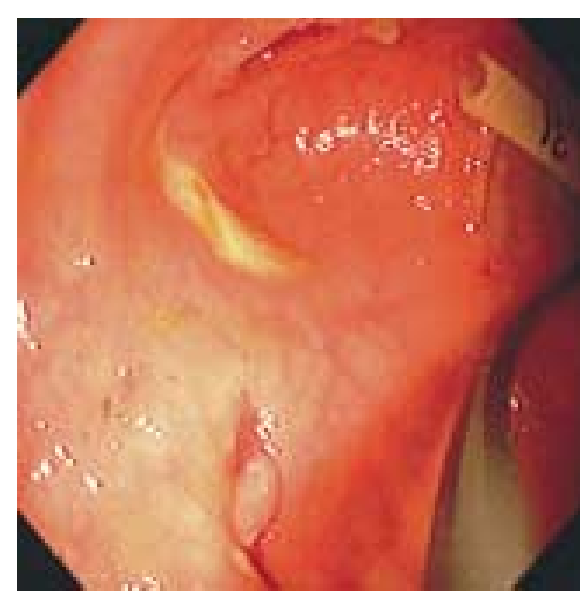

Figure 1 After the external part of the percutaneous endoscopic gastrostomy (PEG) tube had been cut, on the other side, the 7$\mathrm{mm}$ opening of the colocutaneous fistula and the closed gastrocolic fistula with granulation tissue in the transverse colon were noted tulous opening after some deflation of the air. Closure was ascertained by rapid expansion of the colon with air insufflation (Figure 2). At 6 hours after the procedure, the patient was restarted on a liquid diet orally and 3 days later he was discharged without significant sequalae.

Cutaneous-colo-gastric fistula is a rare complication after PEG insertion [1,2]. If a cutaneous-colo-gastric fistula is confirmed, reinsertion of the PEG is not recommended because it might accelerate frank perforation and peritonitis [3]. In the absence of peritonitis, this type of fistula is satisfactorily managed nonsurgically [2]. However, Stefan et al. [4] reported a case of a persistent gastrocolic fistula after the removal of a PEG tube and this fistulous tract was excised by operation. Furthermore, the conservative approach needs several days or weeks for the complete healing of the colocutaneous fistula $[2,5]$, and may delay the start of oral feeding and consequently prolong hospital stay.

As with our patient, endoscopic management using a metallic clip would provide for a more rapid blockage of feculent leak-

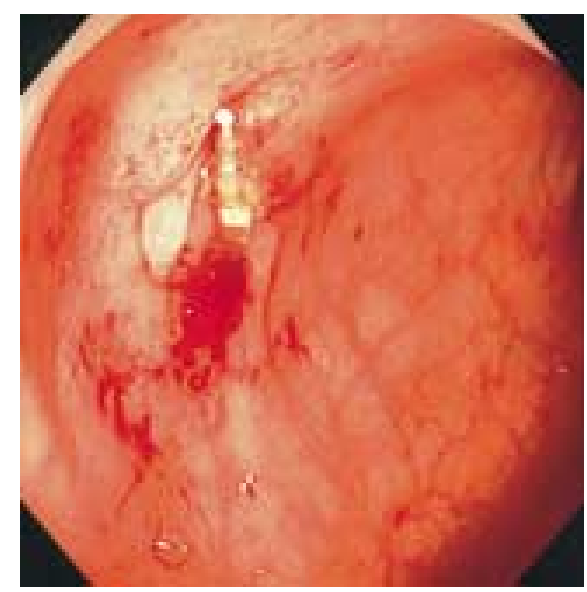

Figure 2 A clip was easily applied to the center of the fistula margin, and successful closure of the colocutaneous fistula was ascertained by rapid expansion of the colon with high-pressure air insufflation age as well as an earlier trial of oral feeding. Therefore, our experience has shown that this endoscopic therapy can be a safe and cost-effective alternative treatment modality for a colocutaneous fistula, especially in patients with a risk of delayed wound healing or infection.

H. S. Kim, D. K. Lee, S. K. Baik, S. O. Kwon Department of Internal Medicine,

Yonsei University Wonju

College of Medicine, Wonju, Korea

\section{References}

${ }^{1}$ Gauderer MW. Pecutaneous endoscopic gastrostomy: a 10-year experience with 220 children. J Pediatr Surg 1991; 26: 288-294

2 Kilmartin C, Brotzman GL, Regan P. Colocutaneous fistula as a complication of PEG tube placement. J Fam Prac 1996; 43: $76-78$

${ }^{3}$ Kinoshita Y, Udagawa H, Kajiyama Y et al. Cologastric fistula and colonic perforation as a complication of percutaneous endoscopic gastrostomy. Surg Laparosc Endosc Percutan Tech 1999; 9: $220-222$

${ }^{4}$ Stefan MM, Holcomb GW III, Ross AJ III. Cologastric fistula as a complication of percutaneous endoscopic gastrostomy. JPEN 1989; 13: $554-556$

${ }^{5}$ Saltzberg DM, Anand K, Juvan P et al. Colocutaneous fistula: an unusual complication of percutaneous endoscopic gastrostomy. JPEN 1987; 11: 86-87

\section{Corresponding Author}

\section{K. Lee, M.D., Ph.D.}

Department of Internal Medicine

Yonsei University Wonju

College of Medicine

Ilsan Dong 162

Wonju 220-701

Korea

Fax: $\quad+82-33-7456782$

E-mail: gidept@wonju.yonsei.ac.kr 